\title{
Weak elastic anisotropy in global seismology
}

by

\author{
Leon Thomsen ${ }^{1,2}$ and Don L. Anderson ${ }^{3}$ \\ ${ }^{1}$ Delta Geophysics, ${ }^{2}$ University of Houston \\ ${ }^{3}$ California Institute of Technology
}

\begin{abstract}
It has been known for over 50 years that seismic anisotropy must be included in a realistic analysis of most seismic data. The evidence for this consists of the observed dependency in many contexts (reviewed briefly here) of seismic velocity upon angle of propagation, and upon angle of S-wave polarization. Despite this well-established understanding, many current investigations continue to employ less realistic isotropic assumptions. One result is the appearance of artefacts which can be interpreted in terms of details of Earth structure, rather than of the restrictive assumptions in the analysis.
\end{abstract}

The reason for this neglect of anisotropy is presumably the greater algebraic complexity, and the larger number of free parameters, of anisotropic seismics. However, the seismic anisotropy in the Earth is usually weak, and the equations for weak anisotropy are only marginally more complex than for isotropy. Further, the additional parameters are commonly required to describe the data. Moreover, the parameters of weak anisotropy defined below (combinations of the anisotropic elastic moduli) are less subject to compounding of uncertainty, and to spatial resolution issues, than are the individual anisotropic moduli themselves. Hence inversions should seek to fit data with these parameters, rather than with those individual moduli. We briefly review the theory for weak anisotropy, and present new equations for the weakly anisotropic velocities of surface waves. The analysis offers new insights on some well-known results found by previous investigations, for example the "Rayleigh wave-Love wave inconsistency", including the facts that Raleigh wave velocities depend not only on the horizontal SV velocity, but also on the anisotropy, and Love wave velocities depend not only on the horizontal SH velocity, but also on the anisotropy. 


\section{INTRODUCTION}

Most of the major features of the structure of Earth's interior were discovered using the concepts of isotropic seismology. However, subtle features require the use of more realistic seismology, based on more realistic rock physics. Although the importance of seismic anisotropy has been known for over 50 years, only in the last decade has the increasing quality and quantity of data forced the recognition that anisotropy is actually crucial for accurate inversions for upper mantle structure. For a time, it was thought that if one considered only "SV"-polarized waves (see theory section, below), one could derive SVwavespeeds without considering the effects of SH- and P- anisotropy. Similarly, it was thought that SH data could be analyzed independently of SV- and P- anisotropy. Neglect of anisotropy, or inappropriate approximations to it, is partly responsible for poor correlation among tomographic models, and for claims of plume sightings in the upper and lower mantles, and for the properties of the upper mantle boundary layer.

Over the last 15 years, there has been increasing refinement of regional and global 3-D seismic models of both $\mathrm{P}$ and $\mathrm{S}$ velocity using a variety of data sets, including absolute travel-times, relative and differential travel-times, surface wave phase and group velocities, diffracted, reflected, and scattered body waves, free oscillations, polarizations, and complete body and surface waveforms. Unhappily, most of these models assume isotropic velocities, and some of the most widely quoted use only relative travel-times of nearly vertically incident teleseismic waves.

By contrast, in exploration geophysics, anisotropic seismics is now the mainstream paradigm; experience over the past 30 years (c.f., e.g., Carcione (2001), Tsvankin et al( 2010)) has shown that it is common that better seismic images, and better subsurface characterization, come from analyzing the data with concepts 
based on anisotropy, rather than isotropy. Recently (11/2014) a major service provider claimed that $80 \%$ of its processing projects were anisotropic.

The main reason why isotropic analysis is still so widely applied in global seismics is presumably the substantially greater algebraic complexity of anisotropic seismology. However, it turns out that, when analyzed properly, seismic anisotropy is only marginally more complicated than seismic isotropy. Of course, there are more elastic parameters to be determined in an anisotropic voxel, but the algebra is only marginally more difficult.

Furthermore, there is commonly a trade-off between spatially complex isotropic structures, and simpler anisotropic ones. The main issue then becomes the necessity and resolvability of the parameters that are used; this is a matter that must be considered separately for each problem, and each dataset.

This paper has four purposes:

- to briefly review the history and theory of anisotropic global seismology (to establish notation, some elementary material is included in an Appendix);

- to show the simplifications offered by the restriction to weak anisotropy, revealing that the anisotropic parameters most commonly appropriate for wave propagation in geophysics are not those defined by Hooke's law, but rather are certain combinations of these;

- to present new equations for the propagation of Rayleigh and Love waves in weakly anisotropic formations, and

- to discuss the rock physics underlying these seismic phenomena.

\section{ANISOTROPY IN THE UPPER MANTLE}

It has been known for 50 years that the uppermost mantle is seismically anisotropic, and that this must be taken into account in the construction of seismic models that approximate well the true Earth structure 
(e.g. Anderson, 1966). Nevertheless, prior to 1989, with few exceptions most seismologists ignored anisotropy. Addressing this was a major motivation for publication of Theory of the Earth (Anderson, 1989), which summarized observations to that point. Anisotropy was taken into account in the spherically symmetric (1D) reference Earth model PREM (Dziewonski \& Anderson, 1981), which requires anisotropy down to a depth of $220 \mathrm{~km}$. When anisotropy is ignored, this biases the depth extent of heterogeneity (e.g. Regan \& Anderson (1984), Anderson and Dziewonski (1982)). Nataf, et al. (1986) were the first ones to include anisotropy in 3-D upper mantle models. They found a belt of Vsv $>$ Vsh around the Pacific, underlying ridges and subduction zones.

Global and regional models of mantle anisotropy have existed since 1984 (e.g., Regan \& Anderson, (1984), Tanimoto \& Anderson (1985), Nataf et al. (1986), Montagner \& Tanimoto (1991)) but there were few subsequent attempts to include anisotropy in global inversions. Nataf et al (1986) and Montagner \& Tanimoto (1991) found systematic variations in shear velocity (polarization variations) depending on the age of the lithosphere down to depths of $\sim 250 \mathrm{~km}$, with Vsv > Vsh under old shields and plate boundaries and Vsh > Vsv under midplate locations and active tectonic belts. Ekstrom and Dziewonski (1998) drew attention to the relatively strong Vsh >VsV anomaly, down to a depth of $200 \mathrm{~km}$, centered near Hawaii. However they, along with many others, inverted Love and Rayleigh waves separately for SH and SV wavespeeds respectively. A good account of this early work is given by Babuska and Cara, 1991). Results to 2002 were summarized by Savage (1999) and by Romanowicz (2003), and numerous papers have appeared since that time.

In these papers, terms like Vsv and Vsh are typically defined as body-wave velocities for horizontally traveling shear waves polarized vertically $\left(\boldsymbol{V}_{\boldsymbol{S} \boldsymbol{V}}\left(90^{\circ}\right)\right)$, and horizontally $\left(\boldsymbol{V}_{\boldsymbol{S H}}\left(90^{\circ}\right)\right)$, respectively $(c f .$, e.g. Eqn. (3) below, and Ekstrom and Dziewonski,1998), although they may be determined from surface waves. The difference between these is indeed an effect of anisotropy, but anisotropic Rayleigh and Love velocities contain other anisotropic effects as well (see the anisotropic surface wave sections below). 
Tanimoto and Anderson (1985) provided maps of shear-wave azimuthal anisotropy at the global scale, showing that the fast axis of shear-wave polarization (see theory sections, below) aligns perpendicular to mid-ocean ridges and parallel to transform faults and inferred plate motions. The use of normal modes and shear-wave splitting has contributed to the explosion of papers dealing with mantle anisotropy in the past 15 years (cf. e.g. Park and Levin, 2002).

Upper mantle radial and azimuthal anisotropy is best resolved using fundamental and higher mode surface waves (Tanimoto \& Anderson (1985), Nataf et al. (1986), Montagner \& Tanimoto (1991), Shapiro and Ritzwoller (2002), Trampert \& Woodhouse (2003), Gung et al.( 2003), Nettles and Dziewonski (2008)). There have been attempts to map transition zone radial (Beghein \& Trampert, 2003) and azimuthal (Trampert \& van Heijst, 2002) S anisotropy, radial S anisotropy in D” (Panning \& Romanowicz, 2004) and P velocity anisotropy in the whole mantle (Boschi \& Dziewonski, 2000; Soldati et al., 2003). Anisotropy in the lower mantle was discussed by Vinnik, et al. (1998). Anisotropy in the inner core was discussed by Song and Richards (1996), and Tromp (2001).

\section{ARTEFACTS INTRODUCED BY THE NEGLECT OF ANISOTROPY}

The persistence of the "plume hypothesis" in seismology, in spite of abundant evidence against it (cf. Anderson and Natland (2014), is partly based on the neglect of anisotropy, sparse ray coverage, and the misuse of Occam's razor. Occam's razor inversion, as applied in seismology, is usually taken to prefer the simplest model, or the smallest and smoothest deviation from a starting model, or the model closest to $a$ priori expectations. This appears to rule out boundary layer models which are heterogeneous on a small scale, anisotropic, or laminated or fractured. However, Anderson and Dziewonski (1982) showed that a given surface wave data set could be equally satisfied by a complex isotopic model or a simple anisotropic model, both involving the same number of parameters. Montagner and Jobert (1988) showed that data for the Indian could be fit by a simple anisotropic model with fewer parameters than a complex isotropic model. 
Shallow mantle heterogeneity and anisotropy, if not included in the analysis, can result in plume-like artefacts, due to "streaking" and "bleeding", respectively. Isotropic inversion of teleseismic near-vertical travel-time datasets (Wolfe et al (2009, 2011); Montelli et al (2004)) suggests the presence of deep vertical zones of low velocity (interpreted as mantle plumes), whereas anisotropic or polarization inversion of data having a wide range of polarizations and directions of approach (Katzman et al. (1998), Collins et al (2012); West et al (2004)) suggest no anomalous low velocity zones, but instead shallow zones of relatively high anisotropy. This raises the possibility that current understanding of many of the subtle features of Earth structure could be erroneous, caused by over-simplified analysis.

The presence of anisotropy in the boundary layer, with a near-vertical low-velocity axis, compounds the problem of vertical streaking of shallow structures into elongated plume-like structures in the deep mantle, which is a well-known artefact of teleseismic travel-time isotropic tomography (Keller, et al (2000), Lei \& Zhou (2006, Figs. 11de).

\section{THE UPPER MANTLE BOUNDARY LAYER}

For decades there has been debate and disagreement about the depth extent of the upper mantle boundary layer, of midocean ridges, and of continental roots, and about the existence and depth extent of lowvelocity features under 'hotspots'. These disagreements can be reconciled by taking into account seismic anisotropy of the type proposed by Kawakatsu et al (2009).

The accurate determination of the elasticity and thickness of the seismic lid (above the Low Velocity Zone) depends on allowing properly for anisotropy. The inferred nature of the lid-LVL boundary also depends on how anisotropic wave propagation and reflections are treated. The interpretation of the seismic velocities in the LVL can be substantially different if anisotropy is ignored. Finally, the effect of the lid and the LVL on teleseismic arrival times has been grossly underestimated by isotropic inversions of body waves (e.g. Montelli et al (2004), Wolfe et al (2009, 2011).

\section{ANISOTROPIC SIMPLIFICATIONS}


Anisotropic seismology inevitably involves simplifications, since the general case (triclinic) requires the determination of 21 independent elasticity stiffness components (see Appendix) in every subsurface voxel, which is normally not feasible. So, assumptions must be made, concerning the symmetry in each voxel, and the orientation of its principal axes. The simplest plausible model is that of polar anisotropy (also known as radial anisotropy, hexagonal symmetry or "Transverse Isotropy, TI" [sic]), with a vertical (radial) symmetry axis; and 5 independent stiffness elements (see Appendix). This case may be justified in terms of sub-seismic structures (layers, crystalline alignment) oriented by gravity, and subjected to equal horizontal stresses.

Beyond such basic assumptions, some simplifications and scaling relations used in in the past are difficult to justify. For example, some do not represent physically realizable structures. (In no physically realizable material can anisotropy be approximated with only 2 parameters, such as Vsh and Vsv.) In some cases, anisotropy is "approximated" by use of only 2 or 3 parameters (rather than the minimum of 5), or sometimes P-wave anisotropy is ignored in analyzing datasets where observed S-wave anisotropy implies that it must be important. In some cases, thinly-layered structures are analyzed with the implausible assumption that the individual layers themselves are intrinsically isotropic, not anisotropic (see rock physics section, below). Some studies use unphysical scaling relations between moduli.

More realistic models assume azimuthal anisotropy (rather than polar anisotropy); the most plausible of these models is orthorhombic (see discussion further below). The model of "Horizontal Transverse Isotropy" $[$ sic $]$ is never physically plausible (see discussion further below), although for vertically incident waves (P and $\mathrm{S}$ ) it is sufficient, since for this restricted dataset, its analysis identical to that of orthorhombic symmetry. If shear- wave splitting (see below) is observed at near-vertical incidence (e.g. West et al, 2009), then there must be corresponding azimuthal effects on P-wave velocities.

Nonetheless, a rational simplification of the exact anisotropic equations is possible. An essential idea making anisotropic seismology feasible is the recognition that, in the Earth, the anisotropy is almost 
always weak, and the anisotropic equations (linearized in appropriately chosen small parameters, see below) are simple enough to be understood intuitively, and computed efficiently. The fact of weak anisotropy is, of course, consistent with the historical success of isotropic seismology in the discovery of the major features of Earth structure.

In the Earth, the seismic anisotropy is almost invariably weak, when defined as a rock property (see below). However, this same weak anisotropy leads to three classes of effects on seismic data:

- Weak effects $\left(2^{\text {nd }}\right.$ order, i.e. relative changes $\left.<<1\right)$ on velocities and travel times, small but necessary to include for understanding subtle features (such as local anomalies and the depth to the LVL);

- Strong effects $\left(1^{\text {st }}\right.$ order, i.e. relative changes $\left.\mathrm{O}(1)\right)$ on reflectivities, wherein the anisotropic terms (although $<<1$ ) are comparable to the isotropic terms (cf. e.g. Thomsen (2014));

- New effects ( $0^{\text {th }}$ order, i.e. not seen at all in isotropic seismics) such as shear-wave splitting.

\section{WEAK POLAR ANISOTROPY; BODY WAVES}

The simplest case of anisotropy that is useful in geophysics has a vertical (radial) pole of rotational elastic symmetry (see Appendix and Theory of the Earth, Chapter 15 (Anderson, 1989)). Although it is not always realistic, it serves well to develop ideas. The wave equation is solved as an eigenvalue equation on a Fourier (plane wave) basis, with 3 eigenvectors (vectors of polarization) and 3 corresponding eigenvalues (velocities) for each direction of propagation. The exact result has been known for over a century; in modern notation it is (e.g. Anderson, 1961):

$$
\begin{aligned}
& \boldsymbol{V}_{\boldsymbol{P}}^{2}(\theta)=\frac{1}{2 \rho}\left[\boldsymbol{C}_{33}+\boldsymbol{C}_{44}+\left(\boldsymbol{C}_{11}-\boldsymbol{C}_{33}\right) \sin ^{2} \theta+\boldsymbol{D}\right] \\
& \boldsymbol{V}_{\boldsymbol{S} V}^{2}(\theta)=\frac{1}{2 \rho}\left[\boldsymbol{C}_{33}+\boldsymbol{C}_{44}+\left(\boldsymbol{C}_{11}-\boldsymbol{C}_{33}\right) \sin ^{2} \theta-\boldsymbol{D}\right]
\end{aligned}
$$




$$
\boldsymbol{V}_{\boldsymbol{S H}}^{2}(\theta)=\frac{1}{\rho}\left[\boldsymbol{C}_{44} \cos ^{2} \theta+\boldsymbol{C}_{66} \sin ^{2} \theta\right]
$$

with

$$
\begin{aligned}
\boldsymbol{D} \equiv\{ & \left(\boldsymbol{C}_{33}-\boldsymbol{C}_{44}\right)^{2}+2\left[2\left(\boldsymbol{C}_{13}+\boldsymbol{C}_{44}\right)^{2}-\left(\boldsymbol{C}_{33}-\boldsymbol{C}_{44}\right)\left(\boldsymbol{C}_{11}+\boldsymbol{C}_{33}-2 \boldsymbol{C}_{44}\right)\right] \sin ^{2} \theta \\
& \left.+\left[\left(\boldsymbol{C}_{11}+\boldsymbol{C}_{33}-2 \boldsymbol{C}_{44}\right)^{2}-4\left(\boldsymbol{C}_{13}+\boldsymbol{C}_{44}\right)^{2}\right] \sin ^{4} \theta\right\}^{1 / 2}
\end{aligned}
$$

The notation for the elastic stiffness matrix elements $\boldsymbol{C}_{\alpha \beta}$ in Eqns. (1) is defined in the Appendix; $\boldsymbol{\theta}$ is the polar angle from the local pole of symmetry, here assumed to be the radial vector. There are 5 independent elastic stiffness elements $\boldsymbol{C}_{\alpha \beta}$ (compared to the two (e.g. $\boldsymbol{\lambda}$ and $\boldsymbol{\mu}$ ) of isotropic seismology). The complexity of the parameter $\boldsymbol{D}$ in Eqn. (1d) is the reason for the difficulty in applying even these simplest anisotropic concepts to real data. Note that equations $(1 \mathrm{a}, \mathrm{b})$ differ only in the algebraic sign of the D-term, so that $\boldsymbol{D}$ constitutes the difference between P-waves and SV-waves.

Since the Fourier basis is complete, the solution to any wave-propagation problem in polar-anisotropic media may be constructed as a sum of plane waves (with differing frequencies and directions of propagation) having these velocities.

In real rocks, the stiffness elements $\boldsymbol{C}_{\alpha \beta}$ are frequency-dependent and complex, leading to dispersive, attenuative wave propagation. However, these issues are beyond the scope of this overview.

Close inspection of Eqns. (1) suggests a re-parameterization of these equations (Thomsen, 1986):

$$
\begin{aligned}
& V_{P 0} \equiv \sqrt{\frac{C_{33}}{\rho}} \quad V_{S 0} \equiv \sqrt{\frac{C_{44}}{\rho}} \\
& \varepsilon \equiv \frac{C_{11}-C_{33}}{2 C_{33}}
\end{aligned}
$$




$$
\begin{aligned}
& \delta \equiv \frac{\left(C_{13}+C_{44}\right)^{2}-\left(C_{33}-C_{44}\right)^{2}}{2 C_{33}\left(C_{33}-C_{44}\right)} \\
& \gamma \equiv \frac{C_{66}-C_{44}}{2 C_{44}}
\end{aligned}
$$

The two velocities in Eqn. (2a) are respectively the vertical (radial) $\mathrm{P}$ and $\mathrm{S}$ velocities. The three nondimensional parameters in Eqns. (2bcd) all reduce to zero in the limiting case of isotropy, and so are direct measures of anisotropy. We may define "weak polar anisotropy" as the case where all of these three parameters are much less than one. Other measures of anisotropy may be appropriate in other cases, but for the body waves of polar anisotropy, these come directly out of the exact equations.

If the exact velocities of Eqns. (1) are linearized in these three small parameters, the result is surprisingly simple (Thomsen (1986), Anderson (1989)):

$$
\begin{aligned}
& \boldsymbol{V}_{\boldsymbol{P}}(\theta) \approx \boldsymbol{V}_{\boldsymbol{P} 0}\left[1+\delta \sin ^{2} \theta \cos ^{2} \theta+\varepsilon \sin ^{4} \theta\right] \\
& \boldsymbol{V}_{\boldsymbol{S} \boldsymbol{V}}(\theta) \approx \boldsymbol{V}_{\boldsymbol{S} 0}\left[1+\left(\frac{\boldsymbol{V}_{\boldsymbol{P} 0}}{\boldsymbol{V}_{\boldsymbol{S} 0}}\right)^{2}(\varepsilon-\delta) \sin ^{2} \theta \cos ^{2} \theta\right] \\
& \boldsymbol{V}_{\boldsymbol{S H}}(\theta)=\boldsymbol{V}_{\boldsymbol{S} 0}\left[1+\gamma \sin ^{2} \theta\right]
\end{aligned}
$$

We make here a few elementary observations arising from Eqns. $(1,3)$, before discussing the specifics of the three modes. These equations show that, to first order, the anisotropic variation of velocity is not governed by the individual $\boldsymbol{C}_{\alpha \beta}$, but rather by the combinations of parameters given in Eqns. (2bcd). Since these combinations govern the seismic data, inversions should seek these combinations in the data, rather than the individual moduli (cf. Chen and Tromp, 2007). Aside from the obvious intuitive accessibility of Eqns. (3), compared to Eqns. (1), there are strong mathematical reasons to use them. The 
partial-derivative kernels which are often used to determine sensitivity of the data to variation in the parameters contain a hidden a priori assumption, which is that the parameters are appropriately chosen, such that partial derivatives make physical sense. But, Eqns. (3) show that, to first order, the individual moduli do not matter; rather it is the combinations (2) which matter (if the individual moduli vary, leaving the combinations (2) unchanged, then the velocities (3) will not change). Hence, partial derivatives should be taken with respect to the five parameters defined in equations (2), holding the other parameters fixed (rather than differentiating with respect to the individual moduli, holding the other moduli fixed).

When the uncertainty which is associated with the individual $\boldsymbol{C}_{\alpha \beta}$ (and which inevitably accompanies any inversion of real data) is propagated in the seismic analysis (using the standard techniques for propagation of uncertainty), it can lead to unacceptable resultant uncertainty of the other $\boldsymbol{C}_{\alpha \beta}$. Hence, it is best to invert instead directly for the combinations which matter, i.e. those in Eqns. (2) (rather than from the individual $\boldsymbol{C}_{\alpha \beta}$, which were defined in the general Hookean constitutive equation (A2), and are not optimal for describing wave propagation.) Following such inversion, the further propagation of uncertainty is minimized.

Further, the spatial resolution of different $\boldsymbol{C}_{\alpha \beta}$ may be different, making analysis of data from the heterogeneous Earth problematic. It obviously makes no sense to deduce values for individual moduli separately (obtaining averages over finite volumes), and then to combine them subsequently into the critical combinations (2), if the finite volumes are different for each modulus, so that each one averages a different portion of the heterogeneous earth. Even if the combination is not performed explicitly by the analyst, it is implicit in the data, since the earth is, in fact, anisotropic. This issue is resolved automatically if the inversion finds the critical parameters (2) directly. 
From Eqn. (3a), the horizontal P-velocity is given by $\boldsymbol{V}_{\boldsymbol{P}}\left(90^{\circ}\right)=\boldsymbol{V}_{\boldsymbol{P} \boldsymbol{0}}(1+\boldsymbol{\varepsilon})$. Since normally $\boldsymbol{\varepsilon}>0$ (c.f. the section on anisotropic rock physics, below), it follows that normally $\boldsymbol{V}_{\boldsymbol{P}}\left(90^{\circ}\right)>\boldsymbol{V}_{\boldsymbol{P O} \text {. At angles }}$ intermediate between vertical and horizontal, the P-velocity variation is not given by simple trigonometric variation between these limiting values, but requires an additional physical parameter, $\boldsymbol{\delta}$.

However, for P-wave problems, only three parameters $\left(\boldsymbol{V}_{\boldsymbol{P O}}, \boldsymbol{\varepsilon}, \boldsymbol{\delta}\right)$ are required, rather than the four $\left(\boldsymbol{C}_{11}, \boldsymbol{C}_{33}, \boldsymbol{C}_{13}, \boldsymbol{C}_{44}\right)$ which are included in the exact Eqns. (1). (Of course, all four of these $\boldsymbol{C}_{\alpha \beta}$ are included within the three essential parameters, but in those combinations (2) which are essential, to first order.) This reduction in the number of free parameters is accomplished by the assumption (easily verified) of weak anisotropy, without the arbitrariness of other simplifications. Of course, most Praypaths are affected by all three parameters.

If $\boldsymbol{\delta}$ is truly small, then Eqn. (2c) may be further linearized:

$$
\delta \rightarrow \delta_{w k} \equiv \frac{C_{13}-\left(C_{33}-2 C_{44}\right)}{C_{33}}
$$

although this does not further simplify the linearized body-wave velocities (3). The use of Eqn. (4) in place of Eqn. (2c) amounts to applying perturbation theory to the exact Eqns. (1) (cf. Montagner and Nataf (1988), and Panning and Nolet (2008)). The full expression (2c) is useful in analyzing some phenomena of strong anisotropy, for example "shear wave triplication", c.f. Thomsen and Dellinger (2003). There is no general rock physics argument determining the algebraic sign of $\delta$, and in analyzing different real datasets, both signs have been inferred by various investigators.

In almost all geophysical contexts, the assumption of weak anisotropy is sufficiently accurate. The approximation embodied in Eqns. (3) is remarkably robust, even when the parameters (2), determined from real data, are not really $<<1$. In any case, the errors may be found exactly (within the assumption of 
polar anisotropy), for any values of the anisotropic parameters, by comparing the "exact" Eqns. (1) with the approximate Eqns. (3).

Note that the parameter

$$
\eta \equiv \frac{\boldsymbol{C}_{11}-2 \boldsymbol{C}_{44}}{\boldsymbol{C}_{13}} \approx 1+\frac{2 \varepsilon-\delta_{w k}}{1-2\left(\boldsymbol{V}_{S 0} / \boldsymbol{V}_{P 0}\right)^{2}}
$$

defined by Anderson (1961) is a function of those parameters (2bcd) which govern the anisotropic variation of velocity to first order. Note that Anderson and Dziewonski (1981) defined a parameter " $\boldsymbol{\eta}^{\text {“ }}$ which is exactly the inverse of that defined above, so some care is required to avoid confusion, when reading the literature. Some studies have determined that $\boldsymbol{\eta}<1$ in particular regions of the subsurface. From Eqn. (5) it is clear that this situation could arise if $\boldsymbol{\delta}_{\boldsymbol{w} \boldsymbol{k}}$ and/or $\boldsymbol{V}_{\boldsymbol{S O} \boldsymbol{O}} / \boldsymbol{V}_{\boldsymbol{P O}}$ were sufficiently large. Neither condition is prohibited by rock physics, but neither seems very plausible.

Alternatively, a determination that $\boldsymbol{\eta}<0$, somewhere within the Earth, might be an artefact, arising from the issues of propagation of uncertainty, and from differing spatial resolution, discussed above. Or, it might arise from the shortcomings of the model of polar anisotropy, i.e. from interpreting azimuthally anisotropic data in terms of polar anisotropy, especially when the input data are azimuthally biased. This possibility can be assessed by examining data residuals for any systematic variation with propagation azimuth.

In either polar anisotropic or azimuthally anisotropic media, two shear waves (with different polarizations) propagate in any direction (at different velocities, in general), $c f$. Eqns. (3bc). It is possible to measure this difference accurately with a single source/receiver pair, so this is the anisotropic signature that is most commonly measured in global seismology. However, such a measurement, of "polarization anisotropy", does not restrict the symmetry class of the medium, unless it is shown to be azimuthally invariant. 
The two polarization directions (for any propagation direction) are properties of the medium, not of the source. If a shear wave is launched into an anisotropic medium (in a given direction) with some other polarization, it does not propagate at all, but rather decomposes (trigonometrically) into the two principal polarization directions. Each of these polarizations propagates at its own velocity (in the general case: $\boldsymbol{V}_{\text {Sfast }}$ or $\boldsymbol{V}_{\text {Sslow }}$ ), which arrive at different times; this is "Shear Wave Splitting". SWS has been discussed extensively by S. Crampin and many others; see Crampin and Peacock (2008) for a recent review of shallow SWS, and Savage (1999) for a recent review of deeper SWS.

In polar anisotropic media, one of the two principal directions of shear polarization is parallel to the planes of symmetry, this is the "SH" mode of Equation (3c). The other is perpendicular to this direction (and almost perpendicular to the direction of propagation). Hence it has (except when the propagation is vertical) a vertical component, and is conventionally called the "SV" mode; $c f$. Equation (3b). These two modes have the same velocity $\boldsymbol{V}_{\boldsymbol{S} \boldsymbol{O}}$ at vertical propagation; at other angles of propagation, their velocities differ, depending on the relative values of the governing parameters in Equations $(3 b, c)$. For horizontal propagation, $\boldsymbol{V}_{\boldsymbol{S} \boldsymbol{V}}\left(90^{\circ}\right)=\boldsymbol{V}_{\boldsymbol{S} \boldsymbol{0}}$ again, whereas $\boldsymbol{V}_{\boldsymbol{S H}}\left(90^{\circ}\right)=\boldsymbol{V}_{\boldsymbol{S} \boldsymbol{\theta}}(1+\boldsymbol{\gamma})$. Since normally $\boldsymbol{\gamma}>0$ (see rock physics discussion below), it is normal that $\boldsymbol{V}_{S \boldsymbol{H}}\left(90^{\circ}\right)>\boldsymbol{V}_{S \boldsymbol{V}}\left(90^{\circ}\right)$, and SWS is most prominent for nearhorizontal propagation, in polar anisotropic media. This applies to body waves; the situation for surface waves is somewhat more complicated (see below).

In heterogeneous anisotropic media, the principal directions of shear-wave polarization vary in space. Hence, as a shear wave (polarized in one of the principal directions) propagates in a given direction, it may refract (following Snell's law) so that, in the new propagation direction, it is no longer polarized along those principal directions. Or, it may enter a new region with different symmetry. In either case, it adjusts its polarization accordingly, by re-splitting, trignonometrically. 
It is clear that complicated arrivals could result, too complicated for this overview. Hence, interpretations of data, especially from curving raypaths, should be careful in the determination of the locus of the splitting (Savage, 1999).

\section{WEAK AZIMUTHAL ANISOTROPY; BODY WAVES}

The case of polar anisotropy, discussed above, serves mainly to fix elementary ideas, and to establish an appropriate strategy for notation. However, most rock formations exhibit lower symmetry than polar anisotropy; this is readily apparent given appropriate datasets. For example, a measurement of polarization anisotropy (as defined above) which varies with azimuth is an immediate indication that the medium is azimuthally anisotropic. Several such cases, are cited above. In such cases, the language of polar anisotropy, e.g. $\boldsymbol{V}_{\boldsymbol{S} \boldsymbol{V}}$ and $\boldsymbol{V}_{\boldsymbol{S H}}$, is misleading, as the principal directions of polarization may be quite different from those of polar anisotropy, except perhaps for certain directions of propagation, such as horizontal propagation, and for certain symmetry classes.

By assumption, at normal incidence in a polar-anisotropic medium, there is no SWS. However, in fact SWS is commonly observed in real data at and near normal incidence, which indicates that the assumption of polar anisotropy is commonly not realized in the Real World. It is easy (in 2014!) to think of physical circumstances which would destroy the azimuthal symmetry of real rock formations, for example oriented cracks (with or without unequal horizontal stresses), or dike emplacement at mid-ocean ridges, or preferential alignment of anisotropic crystals due to flow in the mantle. A controversial discussion of the physical mechanisms that can cause SWS at near-normal incidence in the crust, and their possible geodynamic implications, is given by Crampin and Gao (2013).

Thirty years ago, it became popular to approximate azimuthal anisotropy with the equations of polar anisotropy, rotated $90^{\circ}$, with a horizontal pole of symmetry, so-called "HTI". But, this model physically requires a single set of preferentially aligned vertical fractures (or other flat inclusions) with rotationally invariant compliance ("penny-shaped inclusions") embedded in an otherwise isotropic medium. But, in 
the Earth, the inclusions are seldom rotationally invariant, and the background medium is seldom otherwise isotropic. So, the "HTI" model is seldom an appropriate model to treat real data, and should be consigned to the dustbin of history. Much of the early observations of shear-wave splitting were interpreted with the assumption of HTI symmetry (c.f. Savage (1999) and Crampin and Peacock (2008)), but the increasing quality of data requires a less simplistic analysis.

The case of polar anisotropy with a tilted axis of symmetry (so-called "TTI") is also not physically plausible, since the tectonic forces which cause the tilt presumably also (at shallow depth) introduce oriented fractures, which destroy the rotational symmetry. At greater depth, where the anisotropy is more plausibly caused by partial crystalline alignment caused by flow, the symmetry depends upon the flow itself, and is never plausibly TTI. These physical arguments lessen the reliability of TTI analyses such as those of Montagner and Nataf (1988) and Panning and Nolet (2008).

The simplest plausible case of azimuthal anisotropy is that of orthorhombic symmetry, with one symmetry axis vertical (see Appendix). Formations which are polar-anisotropic, except for a single set of vertical inclusions aligned preferentially in one azimuth, are orthorhombic, whether or not the inclusions are circular. A second set of vertical inclusions, aligned orthogonal to the first, again yields orthorhombic symmetry. Both scenarios are plausible, in simple geologic settings, because of the orthogonality of the stress tensor.

Given a dataset with appropriate azimuthal distribution of raypaths, it is possible to determine the azimuths of the two horizontal principal axes, by straight-forward examination of the azimuthal residuals of the data following an isotropic analysis. It is known (e.g. Tsvankin, 1997) that, along each of these two azimuths, the exact orthorhombic velocities reduce exactly to those of polar anisotropy, Eqns. (1). Of course, it is a different polar system for each of the two principal azimuths. Each of these two may be simplified with the weak anisotropic approximation (3) as discussed above, and appropriate azimuthal subsets of the data may be used to evaluate those parameters $(2,4)$. A $9^{\text {th }}$ ( $\boldsymbol{\delta}$-like) parameter is required 
(Tsvankin, 1997) to complete the orthorhombic characterization, and to analyze data from all the other azimuths.

Lower symmetries (e.g. monoclinic), which are clearly demanded in some contexts, are usually beyond the current state of the art of geophysical analysis. The theory is well-understood (c.f., e.g. Montagner and Nataf (1986), Jech and Psencik (1989), Montagner (2007), Farra and Psencik (2010)), but the application is problematic, since a voxel large enough to encompass enough rays (of varying azimuths and polar angles), in order to characterize the anisotropy, may not be internally homogeneous.

\section{POLAR ANISOTROPIC RAYLEIGH WAVES}

This problem was originally solved by Stoneley (1949). In modern notation, the anisotropic Rayleigh period equation is, from Anderson (1961):

$$
\boldsymbol{R} \equiv-\Gamma_{1} \Pi_{2}+\Gamma_{2} \Pi_{1}=0
$$

where

$$
\begin{aligned}
& \Gamma_{\boldsymbol{i}}=\left[-\gamma_{i} \boldsymbol{v}_{\boldsymbol{i}} \boldsymbol{C}_{33}+\boldsymbol{C}_{13} \boldsymbol{k}\right] \quad \Pi_{\boldsymbol{i}}=\left[\boldsymbol{v}_{\boldsymbol{i}}+\boldsymbol{k} \gamma_{i}\right] \\
& \gamma_{\boldsymbol{i}}=\frac{-\left(\boldsymbol{C}_{44} \boldsymbol{v}_{\boldsymbol{i}}^{2}-\boldsymbol{C}_{11} \boldsymbol{k}^{2}+\rho_{1} \omega^{2}\right)}{\boldsymbol{k} \boldsymbol{v}_{\boldsymbol{i}} \boldsymbol{G}} \\
& \boldsymbol{v}_{1}^{2}=-\frac{\boldsymbol{M}_{1}}{2 \boldsymbol{C}_{33} \boldsymbol{C}_{44}}+\frac{\boldsymbol{M}_{3}}{2 \boldsymbol{C}_{33} \boldsymbol{C}_{44}} \quad \boldsymbol{v}_{2}^{2}=-\frac{\boldsymbol{M}_{1}}{2 \boldsymbol{C}_{33} \boldsymbol{C}_{44}}-\frac{\boldsymbol{M}_{3}}{2 \boldsymbol{C}_{33} \boldsymbol{C}_{44}} \\
& \boldsymbol{M}_{3}^{2}=\left[\boldsymbol{M}_{1}^{2}-4 \boldsymbol{M}_{2} \boldsymbol{C}_{33} \boldsymbol{C}_{44}\right] \\
& \boldsymbol{M}_{1}=\boldsymbol{C}_{33}\left(\rho_{1} \omega^{2}-\boldsymbol{C}_{11} \boldsymbol{k}^{2}\right)+\boldsymbol{C}_{44}\left(\rho_{1} \omega^{2}-\boldsymbol{C}_{44} \boldsymbol{k}^{2}\right)+\boldsymbol{k}^{2}\left(\boldsymbol{C}_{13}+\boldsymbol{C}_{44}\right)^{2} \\
& \boldsymbol{M}_{2}=\left(\rho_{1} \omega^{2}-\boldsymbol{C}_{11} \boldsymbol{k}^{2}\right)\left(\rho_{1} \omega^{2}-\boldsymbol{C}_{44} \boldsymbol{k}^{2}\right)
\end{aligned}
$$


with $\boldsymbol{k}$ the wavenumber, and $\boldsymbol{\omega}$ the angular frequency. Eqn. (6), with its layers (7) of notation, while exact within its assumptions (linear elasticity, polar anisotropy, half-space) is difficult to understand intuitively, and in computation, it functions as a black box.

However, if we use the parameters in Eqns. $(2,4)$, and assume weak anisotropy, Eqn. (6) reduces to:

$$
\boldsymbol{R} \approx\left[\left(k^{2}+v_{20}^{2}\right)^{2}-4 k^{2} v_{10} v_{20}\right]+\delta_{w k} \boldsymbol{R}_{\delta}+\varepsilon \boldsymbol{R}_{\varepsilon} \approx 0
$$

where

$$
\begin{aligned}
& v_{10}^{2}=\boldsymbol{k}^{2}-\frac{\omega^{2}}{\boldsymbol{V}_{\boldsymbol{P} 0}^{2}} \quad \boldsymbol{v}_{20}^{2}=\boldsymbol{k}^{2}-\frac{\omega^{2}}{\boldsymbol{V}_{\boldsymbol{S} 0}^{2}} \\
& \boldsymbol{R}_{\delta}=\left[\frac{4 \boldsymbol{C}_{33} k^{4} v_{10} v_{20}}{\rho \omega^{2}}+\frac{\left(k^{2}+v_{20}^{2}\right) \boldsymbol{C}_{33}^{2}}{\left(\boldsymbol{C}_{33}-\boldsymbol{C}_{44}\right) \rho \omega^{2}}\left(k^{2}\left(2 v_{20}^{2}-\frac{\boldsymbol{C}_{44}}{\boldsymbol{C}_{33}}\left(k^{2}+v_{20}^{2}\right)\right)-\frac{v_{10}^{2} \rho \omega^{2}}{\boldsymbol{C}_{44}}\right)\right] \\
& \boldsymbol{R}_{\varepsilon}=\left[\frac{4 \boldsymbol{C}_{33} k^{6} v_{20}}{\rho \omega^{2} v_{10}}-\frac{\left(k^{2}+v_{20}^{2}\right) k^{2} \boldsymbol{C}_{33}^{2}}{\left(\boldsymbol{C}_{33}-\boldsymbol{C}_{44}\right) \rho \omega^{2}}\left(\left(2 v_{20}^{2}-\frac{\boldsymbol{C}_{44}}{\boldsymbol{C}_{33}}\left(k^{2}+v_{20}^{2}\right)\right)-\frac{\rho \omega^{2}}{\boldsymbol{C}_{44}}\right)\right]
\end{aligned}
$$

In Eqn. (8), the special case of isotropy is just the case with the last two terms set to zero (Anderson, 1961). The anisotropic variation is then given, to first order, when these two terms are included. This equation is a fourth-order equation in the unknown $\boldsymbol{k}^{2}$, which does have a closed-form, albeit complicated, algebraic solution.

However, a more intuitive result follows from the observation that the Rayleigh wave phase velocity $\boldsymbol{V}_{\boldsymbol{R}}$ is expected to be somewhat less than the shear-wave body velocity $\boldsymbol{V}_{\boldsymbol{S O} \boldsymbol{O}}$. Hence we define a small quantity $\zeta$

$$
\frac{\omega}{k} \equiv V_{R} \equiv V_{S 0}(1-\zeta)
$$

and further linearize Eqn. (9) in $\boldsymbol{\zeta}$.. The result is a simple expression for the Rayleigh velocity $\boldsymbol{V}_{\boldsymbol{R}}$ : 


$$
\boldsymbol{V}_{\boldsymbol{R}} \approx \boldsymbol{V}_{\boldsymbol{S} 0}\left\{1-\frac{1}{16\left(1-2 \boldsymbol{V}_{\boldsymbol{S} 0}^{2} / \boldsymbol{V}_{\boldsymbol{P} 0}^{2}\right)}\left[1+\frac{2}{\left(1-\boldsymbol{V}_{\boldsymbol{S} 0}^{2} / \boldsymbol{V}_{\boldsymbol{P} 0}^{2}\right)}\left(\varepsilon+\left(\varepsilon-\delta_{w \boldsymbol{k}}\right) \frac{\boldsymbol{V}_{\boldsymbol{P} 0}^{2}}{\boldsymbol{V}_{\boldsymbol{S} 0}^{2}}\right)\right]\right\}
$$

The leading term on the right of this expression is the classical isotropic result; the last term shows clearly how the weak anisotropy affects the Rayleigh phase velocity. It is clear that a measurement of the Rayleigh velocity $\boldsymbol{V}_{\boldsymbol{R}}$ does not allow a determination of $\boldsymbol{V}_{\boldsymbol{S}_{0}}=\sqrt{\boldsymbol{C}_{44} / \rho}$ without a concurrent determination of the anisotropy parameters $\boldsymbol{\varepsilon}$ and $\boldsymbol{\delta}_{\mathrm{wk}}$. This result is consistent, of course, with the PREM calculations of (Dziewonski \& Anderson, 1981), which showed the sensitivity of Rayleigh waves to the $\boldsymbol{\eta}$ parameter (5), in addition to $\boldsymbol{V}_{\boldsymbol{S O}}$.

Note in particular that, if the Rayleigh velocity $\boldsymbol{V}_{\boldsymbol{R}}$ is anomalously large, this could be attributed to the anisotropy parameters, or to the $\boldsymbol{V}_{\boldsymbol{P O}} / \boldsymbol{V}_{\boldsymbol{S O}}$ ratio, without concluding that $\boldsymbol{V}_{\boldsymbol{S O}}=\boldsymbol{V}_{\boldsymbol{S} V}\left(\mathbf{9 0}^{\circ}\right)$ is greater than $V_{S H}\left(\mathbf{9 0}^{\circ}\right)=V_{S O}(1+\gamma)$, i.e. without concluding that $\gamma<0$. However, the anisotropic correction terms normally decrease $\boldsymbol{V}_{\boldsymbol{R}}$ (see the rock physics section below), so the physical cause of an anomalously large $\boldsymbol{V}_{\boldsymbol{R}}$ should be sought in the ratio $\boldsymbol{V}_{\boldsymbol{P O}} / \boldsymbol{V}_{\boldsymbol{S O}}$, or in a failure of the assumption of vertical polar anisotropy. Note further that both the (near-horizontal) P-wave anisotropy $\boldsymbol{\varepsilon}$, and the SV anisotropy $\left(\boldsymbol{\varepsilon}-\boldsymbol{\delta}_{\boldsymbol{w} \boldsymbol{k}}\right)\left(\boldsymbol{V}_{\boldsymbol{P} \boldsymbol{0}} / \boldsymbol{V}_{\boldsymbol{S O}}\right)^{2}$ appear in Eqn. (11).

Eqns. (6-11) apply to the case of a uniform polar anisotropic half-space (hence there is no frequency dependence). For applications to the real Earth, with vertical (and horizontal) variations of elastic properties, they have to be generalized, in ways that are similar to the corresponding isotropic problem. This generalization inevitably introduces characteristic thicknesses, which render the result dispersive (Park, 1996).

The analysis above is for polar anisotropy. An elegant treatment of surface waves in azimuthally anisotropic media, again using the assumption of weak anisotropy, is given by Smith and Dahlen (1973). 
However, their analysis leaves implicit the connection between the surface wave velocities and the anisotropy of P- and S- body waves. Further, they assume that the polarizations of surface waves are horizontal and vertical, respectively; this restricts the validity of their analysis to anisotropic systems with a horizontal plane of symmetry. Montagner and Nataf (1986) follow a similar approach, and provide a more comprehensive description of the effect of general weak anisotropy on surface waves and its relationship with body wave anisotropy.

\section{POLAR ANISOTROPIC LOVE WAVES}

Following Anderson (1961), the exact equation for the Love wave phase velocity for a polar anisotropic layer of thickness $2 \boldsymbol{H}$ (with elastic parameters $\boldsymbol{C}_{44}^{\boldsymbol{H}}, \boldsymbol{C}_{66}^{\boldsymbol{H}}$ and $\beta_{\boldsymbol{H}}^{2} \equiv \boldsymbol{C}_{44}^{\boldsymbol{H}} / \rho_{\boldsymbol{H}}=\boldsymbol{C}_{66}^{\boldsymbol{H}} /\left(1+2 \gamma_{\boldsymbol{H}}\right) \rho_{\boldsymbol{H}}$ )

over a polar anisotropic half-space (with elastic parameters $\boldsymbol{C}_{44}, \boldsymbol{C}_{66}$ and $\left.\beta^{2} \equiv C_{44} / \rho=C_{66} /(1+2 \gamma) \rho\right)$ is:

$\tan \left[2 \boldsymbol{k H} \sqrt{\frac{\boldsymbol{C}_{66}^{\boldsymbol{H}}}{\boldsymbol{C}_{44}^{\boldsymbol{H}}}} \sqrt{\frac{\boldsymbol{V}_{\boldsymbol{L}}^{2}}{\beta_{\boldsymbol{H}}^{2}}-1}\right]=\sqrt{\frac{\boldsymbol{C}_{44} \boldsymbol{C}_{66}}{\boldsymbol{C}_{44}^{\boldsymbol{H}} \boldsymbol{C}_{66}^{\boldsymbol{H}}}} \sqrt{\left(1-\frac{\boldsymbol{V}_{\boldsymbol{L}}^{2}}{\beta^{2}}\right) /\left(\frac{\boldsymbol{V}_{\boldsymbol{L}}^{2}}{\beta_{\boldsymbol{H}}^{2}}-1\right)}$

The velocity parameters $\boldsymbol{\beta}_{\boldsymbol{H}}$ and $\boldsymbol{\beta}$ are just the vertical shear body-velocities $\boldsymbol{V}_{\boldsymbol{S} \boldsymbol{O}}$, defined in Eqns. (2), of the upper layer and half-space. However, since the Love wave travels horizontally, polarized horizontally, it may be more instructive to write the equation in terms of the corresponding stiffness element $\boldsymbol{C}_{\boldsymbol{6} \boldsymbol{6}}$, and $\boldsymbol{V}_{\boldsymbol{S} \boldsymbol{0}}$, for both the upper layer and the lower half-space:

$\tan \left[2 \boldsymbol{k} \boldsymbol{H} \sqrt{\frac{\boldsymbol{C}_{66}^{\boldsymbol{H}}}{\boldsymbol{C}_{66}}} \sqrt{\left(\frac{\boldsymbol{V}_{\boldsymbol{L}}}{\boldsymbol{V}_{\boldsymbol{S} 0}^{\boldsymbol{H}}}\right)^{2}-1}\right]=\frac{\boldsymbol{V}_{\boldsymbol{S} 0}}{\boldsymbol{V}_{\boldsymbol{S} 0}^{\boldsymbol{H}}} \sqrt{\frac{\rho \boldsymbol{C}_{66}}{\rho_{\boldsymbol{H}} \boldsymbol{C}_{66}^{\boldsymbol{H}}}} \sqrt{\left(1-\left(\frac{\boldsymbol{V}_{\boldsymbol{L}}}{\boldsymbol{V}_{\boldsymbol{S} 0}}\right)^{2}\right) /\left(\left(\frac{\boldsymbol{V}_{\boldsymbol{L}}}{\boldsymbol{V}_{\boldsymbol{S} 0}^{\boldsymbol{H}}}\right)^{2}-1\right)}$ 
Since, this expression implicitly contains the anisotropy parameter $\gamma$, for both layer and half-space. Further simplification would now be possible (taking advantage of the smallness of these anisotropy parameters), by adopting further assumptions on $\boldsymbol{k}, \boldsymbol{H}$, and $\boldsymbol{C}_{66} / \boldsymbol{C}_{66}^{\boldsymbol{H}}$, suitable to some particular context in the Earth. Even without examining such a specific case, Equation (13) shows that observation of the Love wave velocity $\boldsymbol{V}_{\boldsymbol{L}}$ does not permit a direct determination of $\boldsymbol{C}_{\boldsymbol{\sigma} \boldsymbol{6}}$, either in or below the layer $\boldsymbol{H}$, without a concurrent determination of the corresponding anisotropy parameters $\gamma$.

The corresponding isotropic equation is

$$
\tan \left[2 \boldsymbol{k} \boldsymbol{H} \sqrt{\frac{\mu_{\boldsymbol{H}}}{\mu}} \sqrt{\frac{\rho_{\boldsymbol{H}} \boldsymbol{V}_{\boldsymbol{L}}^{2}}{\mu_{\boldsymbol{H}}}-1}\right]=\frac{\mu}{\mu_{\boldsymbol{H}}} \sqrt{\left(1-\frac{\rho \boldsymbol{V}_{\boldsymbol{L}}^{2}}{\mu}\right) /\left(\frac{\rho_{\boldsymbol{H}} \boldsymbol{V}_{\boldsymbol{L}}^{2}}{\mu_{\boldsymbol{H}}}-1\right)}
$$

Eqn. (14) shows clearly that it is not an acceptable approximation to invert Love wave data from anisotropic formations using isotropic algorithms, which do not include the anisotropy parameters implicit in Eqn. (13). Because the Rayleigh wave Eqn. (11) and the Love wave Eqn. (13) together contain all 5 elastic parameters, the joint interpretation of measurements of $\boldsymbol{V}_{\boldsymbol{R}}$ and $\boldsymbol{V}_{\boldsymbol{L}}$ necessarily involves estimation of all 5 parameters, not just 1 or 2.

\section{ANISOTROPIC FREE OSCILLATIONS}

Inversion of the periods of free oscillation for anisotropic elastic properties is inherently an intensive computation, whether the anisotropy is weak, or not. However, since the anisotropy is weak, it is more appropriate to invert for the anisotropic parameters $(2,4)$ directly than for the individual $\boldsymbol{C}_{\alpha \beta}$, since the anisotropic effect is given by the combinations of $\boldsymbol{C}_{\alpha \beta}$ contained within the parameters, rather than by the individual $\boldsymbol{C}_{\alpha \beta}$ themselves. Eqns. (3) show that a partial derivative of the velocities (with respect to one stiffness component, with the others held constant) does not make sense, since it is the combinations $(2,4)$ 
which control the data. The appropriate partial derivative is done with respect to one of the anisotropy parameters $(2,4)$, with the others held constant. This applies also to the periods of free oscillation.

The application of this strategy is straightforward; one simply replaces, in the standard equations (cf., e.g. Dziewonski \& Anderson, 1981 (App.)) for free oscillations in a radially anisotropic earth, the $\boldsymbol{C}_{\alpha \beta}$ with:

$$
\begin{aligned}
& C_{11} \rightarrow C_{33}(1+2 \varepsilon) \\
& C_{13} \rightarrow C_{33}-2 C_{44}+\delta_{w k} C_{33} \\
& C_{66} \rightarrow C_{44}(1+2 \gamma)
\end{aligned}
$$

and inverts for the anisotropic parameters directly. It is to be expected that the resolution kernels for the small anisotropic parameters will be more spatially extensive than those for the vertical velocities $\boldsymbol{V}_{\boldsymbol{P} \boldsymbol{0}}$ and $\boldsymbol{V}_{\boldsymbol{S} \boldsymbol{0}}$. A more general treatment is given by Romanowicz and Snieder (1988).

For more realistic modelling of anisotropy, one should follow the strategy described by Tsvankin (1997) for evaluating the parameters of orthorhombic anisotropy.

\section{ANISOTROPIC ROCK PHYSICS}

Independent of the discussion above of anisotropic wave propagation are a number of ideas of anisotropic rock physics, dealing with the sub-seismic features in the rock mass which create the larger-scale anisotropy. The causes of anisotropy may be intrinsic (e.g. preferred orientations of minerals or cracks, or simply of stress) or extrinsic (preferred orientation of larger (but still not seismically resolvable) features such as layers, horizontal or dipping). In the latter case, it has long been understood ( $c f$. Backus (1962)) that a layered medium (with stationary statistics of the elastic variations among the various layers) propagates long-wavelength elastic waves as though it were a uniform polar anisotropic medium, if the individual layers themselves are isotropic or polar anisotropic.

A layered elastic medium which conforms to Backus' assumptions also exhibits anisotropic apparent attenuation; a purely elastic effect which is outside the scope of the present paper. Heterogeneity not 
conforming to Backus' assumptions must be handled by an assumption of large-scale piecewise homogeneity, or via the equation of motion for inhomogeneous media ( $c f$. Appendix).

The assumption of plausible mineral assemblages and fabrics allows one to constrain the relations between various anisotropic parameters (e.g. Nataf et al (1986); Montagner and Anderson (1989ab); Kawakatsu et al (2009); Anderson (2011)), subject to the validity of the assumption. An important point is that if the anisotropy is in fact extrinsic, then it is not subject to mineralogic assumptions alone, but requires assumptions about the larger-scale (but still sub-seismic) features which create the anisotropy extrinsically. It is clear that this mineralogic approach is not likely to lead to definitive conclusions. (See also Song and Kawakatsu (2012), Wang, et al (2013).)

To illustrate the point, we consider further here the case of both extrinsic and intrinsic polar anisotropy. First, if the individual layers are isotropic, and if the elastic variations among the layers are small, then Backus' equations may be linearized in these small variations, and recast in terms of the anisotropic parameters of Eqns. (2, 4) (Thomsen, 2014):

$$
\begin{aligned}
& \varepsilon_{\text {thin-isolyrs }}=2\left[\langle\mu\rangle\left\langle\boldsymbol{M}^{-1}\right\rangle-\langle\mu / \boldsymbol{M}\rangle\right]+2\left[\langle\mu / \boldsymbol{M}\rangle^{2}-\left\langle\mu^{2} / \boldsymbol{M}\right\rangle\left\langle\boldsymbol{M}^{-1}\right\rangle\right] \\
& \delta_{\text {thin-isolyrs }}=2\left\langle\mu^{-1} \Delta(\mu / \boldsymbol{M})\right\rangle \frac{(1-\langle\mu / \boldsymbol{M}\rangle)}{\left(\left\langle\mu^{-1}\right\rangle-\left\langle\boldsymbol{M}^{-1}\right\rangle\right)} \\
& \gamma_{\text {thin-isolyrs }}=\frac{1}{2}\left[\langle\mu\rangle\left\langle\mu^{-1}\right\rangle-1\right]
\end{aligned}
$$

where $\boldsymbol{M}=\boldsymbol{K}+4 \boldsymbol{\mu} / \mathbf{3}$ is the longitudinal modulus. Here, the angle brackets $<.>$ indicate thicknessweighted averages (across the thin-layered sequence) of the layer property indicated within the brackets, and the symbol $\Delta \boldsymbol{x}$ indicates a local deviation from the average value, $\langle\boldsymbol{x}\rangle$. The mathematical form of Eqn. (16c) requires (according to the Cauchy-Schwarz inequality) that $\boldsymbol{\gamma}_{\text {thin-isolyrs }}$ is non-negative. With 
well-known correlations between the longitudinal modulus $\boldsymbol{M}$ and the shear modulus $\boldsymbol{\mu}$, it follows that $\boldsymbol{\varepsilon}_{\text {thin-isolyrs }}$, Eqn. (16b), is also normally positive, by the same mathematical theorem.

However, there is no similar argument for $\boldsymbol{\delta}_{\text {thin-isolyrs }}$, Eqn. (16a), and in fact different real datasets yield both positive and negative values of $\boldsymbol{\delta}$. The crucial term in Eqn. (16a) is the leading term, involving the deviations $\Delta(\mu / M)=\Delta\left(\left(V_{S} / V_{P}\right)^{2}\right)$ within the layered sequence. Since $\left(V_{S} / V_{P}\right)$ usually does not vary markedly in a layered sequence, it is tempting to set this ratio as a constant, which leads to $\boldsymbol{\delta}_{\text {thin-isolyrs }}=0$, which is a special case of no particular interest. Depending on the statistics of $\Delta(\mu / M)$, the computed parameter $\boldsymbol{\delta}_{\text {thin-isolyrs }}$ may be either positive or negative.

However, an important point is that Eqns. (16) ignore the fact that the individual layers may themselves be anisotropic, intrinsically. In this case, Eqns. (16) must be generalized (Thomsen, 2014) to:

$$
\begin{aligned}
& \varepsilon_{\text {thin-lyrs }}=\varepsilon_{\text {thin-isolyrs }}+\left\langle(\varepsilon-\delta) \boldsymbol{C}_{33}+2 \delta \boldsymbol{C}_{44}\right\rangle\left\langle\boldsymbol{C}_{33}^{-1}\right\rangle+\langle\delta\rangle\left(1-2\left\langle\boldsymbol{C}_{44} \boldsymbol{C}_{33}^{-1}\right\rangle\right) \\
& \delta_{\text {thin-lyrs }}=\delta_{\text {thin-isolyrs }}=+\langle\delta\rangle\left[\frac{2\left(\left\langle 1-\boldsymbol{C}_{44} \boldsymbol{C}_{33}^{-1}\right\rangle\right)}{\left(1-\left\langle\boldsymbol{C}_{33}^{-1}\right\rangle /\left\langle\boldsymbol{C}_{44}^{-1}\right\rangle\right)}-1\right] \\
& \gamma_{\text {thin-lyrs }}=\gamma_{\text {thin-isolyrs }}+\left\langle\gamma \boldsymbol{C}_{44}\right\rangle\left\langle\boldsymbol{C}_{44}^{-1}\right\rangle
\end{aligned}
$$

In most cases, the values of the sub-seismic anisotropic parameters which appear above on the right must be assumed, since determination from data is not usually feasible. This naturally restricts the validity of the conclusions which follow such assumptions. 
The rock physics of the parameters of azimuthal anisotropy is still more ambiguous. Azimuthal anisotropy may be caused by distributions of aligned cracks, by unequal horizontal stresses, by processes (e.g. dike formation) at mid-oceanic ridges, or to flow in the mantle. Each of these causes, and several others less obvious, may be more important in some zones of the Earth, and less important in others. For example, the occurrence of oriented fractures is not subject to doubt at shallow depths, but becomes less plausible at great depth; the transition between these domains is subject to continuing debate, outside the scope of this paper. As another example, the preferred orientation of anisotropic crystals, caused by flow in the mantle (or within salt bodies within the upper crust), leads of course to anisotropy (c.f. Savage (1999)), with symmetry that depends upon the flow itself.

However, an important point is that the seismic analysis discussed further above may be conducted independently of any rock-physics analysis, which describes the sub-seismic features which ultimately cause the anisotropy. The rock physics analysis properly follows the seismic analysis, rather than preceding it, or accompanying it.

\section{APPENDIX: THE ANISOTROPIC ELASTIC STIFFNESS TENSOR}

The equation of motion for an elastic continuum is

$$
\rho \frac{\partial^{2} \boldsymbol{u}_{\boldsymbol{i}}}{\partial \boldsymbol{t}^{2}}=\frac{\partial \tau_{\boldsymbol{i j}}}{\partial \boldsymbol{x}_{\boldsymbol{j}}}
$$

where $\boldsymbol{\rho}$ is density, $\boldsymbol{u}_{\boldsymbol{i}}$ is a component of displacement, $\boldsymbol{t}$ is time, $\boldsymbol{\tau}_{\boldsymbol{i} \boldsymbol{j}}$ is a component of stress, and $\boldsymbol{x}_{\boldsymbol{j}}$ is a component of position. Repeated indices imply a sum (1 to 3$)$. The stress is linearly related to the strain $\boldsymbol{e}_{\boldsymbol{k}}$ by Hooke's law:

$$
\tau_{i j}=C_{\partial j k l} e_{k l}=C_{\partial j k l} \frac{1}{2}\left(\frac{\partial \boldsymbol{u}_{k}}{\partial \boldsymbol{x}_{l}}+\frac{\partial \boldsymbol{u}_{l}}{\partial \boldsymbol{x}_{k}}\right)
$$


where $\boldsymbol{C}_{i j k \boldsymbol{l}}$ is the anisotropic elastic stiffness tensor. Substituting Eqn. (A2) into (A1), recognizing the symmetry of stress and of strain, and assuming that the medium is uniform on a scale comparable to a wavelength, yields the anisotropic wave equation:

$$
\frac{\partial^{2} \boldsymbol{u}_{i}}{\partial \boldsymbol{t}^{2}}=\left(\frac{\boldsymbol{C}_{i j k \boldsymbol{l}}}{\rho}\right) \frac{\partial^{2} \boldsymbol{u}_{\boldsymbol{l}}}{\partial \boldsymbol{x}_{j} \partial \boldsymbol{x}_{\boldsymbol{k}}}
$$

Eqn. (A3) is written in Cartesian coordinates; it may be transformed by standard vector methods to spherical polar coordinates, for application to global problems. What follows is a brief discussion of the implications of this equation; for a fuller discussion of anisotropic seismology, see the monographs by Tsvankin (2012) or Carcione (2001).

The elastic properties of the medium are contained within the (rank 4, dimension 3) stiffness tensor $\boldsymbol{C}_{\boldsymbol{i j k} \boldsymbol{l}}$, which is famously hard to visualize. Fortunately, due to its symmetries, it may be displayed (Voigt, 1910) as a matrix (of rank 2, dimension 6) $\boldsymbol{C}_{\alpha \beta}$, following the pairwise recipe:

$$
\begin{aligned}
& \ddot{i j}=\boldsymbol{j i}=11 \quad 22 \quad 33 \quad 23 \quad 13 \quad 12
\end{aligned}
$$

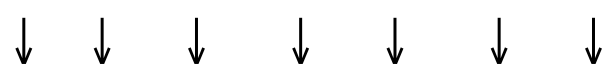

$$
\begin{aligned}
& \alpha=1 \quad 2 \quad 3 \quad 4 \quad 5 \quad 6
\end{aligned}
$$

(The tensor $\boldsymbol{C}_{i j k l}$ should be used for all calculations, since it rotates "like a tensor"; the matrix $\boldsymbol{C}_{\alpha \beta}$ should be used for display and discussion only.) The most general anisotropy (tri-clinic) has 21 independent elements in the symmetric matrix $\boldsymbol{C}_{\alpha \beta}$; such low symmetries are usually not feasible to analyze in geophysics. The highest symmetry is isotropic, in which case the matrix simplifies: 


$$
\mathscr{C}_{\text {iso }}=\left[\begin{array}{cccccc}
\boldsymbol{M} & \lambda & \lambda & & & \\
& \boldsymbol{M} & \lambda & & & \\
& & \boldsymbol{M} & & & \\
& & & \mu & & \\
& & & & \mu & \\
& & & & & \mu
\end{array}\right]
$$

where the lower triangle of this symmetric matrix is omitted, for simplicity. In the upper triangle, the blank elements are zero. The longitudinal modulus $\boldsymbol{M}=\boldsymbol{K}+4 \mu / 3=\rho \boldsymbol{V}_{\boldsymbol{P}}^{2}$ appears in 3 places, indicating invariance with direction, i.e. isotropy. Likewise, the shear modulus $\mu=\rho V_{S}^{2}$ appears in 3 places. The computed Lame parameter $\lambda \equiv \boldsymbol{M}-2 \mu$ is also shown explicitly for compactness.

The simplest case of anisotropy which is of use in geophysics is that of polar anisotropy, wherein the medium has a vertical (radial) pole of rotational symmetry This case used to be called Transverse Isotropy (Love, 1928), but this name has confused generations of students, with the word "Isotropy" used to label a type of Anisotropy.

For polar anisotropy, the elastic stiffness matrix has the form:

$$
\mathscr{C}_{\text {polar }}=\left[\begin{array}{cccccc}
\boldsymbol{C}_{11} & \left(\boldsymbol{C}_{11}-2 \boldsymbol{C}_{66}\right) & \boldsymbol{C}_{13} & & & \\
& \boldsymbol{C}_{11} & \boldsymbol{C}_{13} & & & \\
& & C_{33} & & & \\
& & & C_{44} & & \\
& & & & C_{44} & \\
& & & & & C_{66}
\end{array}\right]
$$


Of course, the stiffness matrix (A6) is referred to the principal coordinate system of the medium, which most plausibly has its polar symmetry axis $(\boldsymbol{i}=3)$ aligned with the radial vector. There are five independent parameters: $\boldsymbol{C}_{11}, \boldsymbol{C}_{33}, \boldsymbol{C}_{13}, \boldsymbol{C}_{44}$, and $\boldsymbol{C}_{66}$. (These were termed A, C, F, L, and N, respectively, by Love (1928), and this notation is still occasionally seen today, although it offers no advantage over the matrix notation used here.) The use of Eqn. (A6) in (A3), assuming a plane-wave (Fourier) basis results in an eigenvalue equation for the three plane-wave eigenvectors (polarizations) and eigenvalues (velocities); these are given in Eqns. (1) of the main text.

From those equations (1), it is clear that $\boldsymbol{C}_{11}=\boldsymbol{C}_{22}=\rho \boldsymbol{V}_{\boldsymbol{P}}\left(90^{\circ}\right)^{2}$ controls the horizontal P-velocity; the equality of these two moduli is a result of the assumption of symmetry about the vertical pole. Similarly, $C_{33}=\rho V_{\boldsymbol{P}}\left(0^{\circ}\right)^{2}$ controls the vertical P-velocity. $\boldsymbol{C}_{44}=\boldsymbol{C}_{55}=\rho \boldsymbol{V}_{\boldsymbol{S V}}\left(0^{\circ}\right)^{2}=\rho \boldsymbol{V}_{\boldsymbol{S H}}\left(0^{\circ}\right)^{2}$ controls the vertical shear velocity, independent of polarization. $\boldsymbol{C}_{\boldsymbol{\sigma} \boldsymbol{\sigma}}=\rho \boldsymbol{V}_{\boldsymbol{S} \boldsymbol{H}}\left(90^{\circ}\right)^{2}$ controls the horizontal shear velocity, with horizontal polarization. (The horizontal shear velocity with vertical polarization, $\boldsymbol{V}_{\boldsymbol{S V}}\left(90^{\circ}\right)$, is the same as $\boldsymbol{V}_{\boldsymbol{S} V}\left(0^{\circ}\right)$.) $\boldsymbol{C}_{\boldsymbol{1 3}}=\boldsymbol{C}_{23}$ affects the velocities $\boldsymbol{V}_{\boldsymbol{P}}(\boldsymbol{\theta})$ and $\boldsymbol{V}_{\boldsymbol{S} V}(\boldsymbol{\theta})$ at intermediate polar angles $\boldsymbol{\theta}$. $\boldsymbol{C}_{\boldsymbol{1 2}}$ is computed from elements already defined, by the formula indicated above.The simplest realistic case of azimuthal anisotropy is that of orthorhombic anisotropy (more properly: orthotropic). It has the symmetry of a brick, with 9 independent parameters:

$$
\mathscr{C}_{\text {ortho }}=\left[\begin{array}{llllll}
C_{11} & C_{12} & C_{13} & & & \\
& C_{22} & C_{23} & & & \\
& & C_{33} & & & \\
& & & C_{44} & & \\
& & & & C_{55} & \\
& & & & & C_{66}
\end{array}\right]
$$

This seems to be quite intimidating, and infeasible in geophysics, but in fact it may be handled by appropriate re-parameterization of the elasticity, and parsing of the data (cf. Tsvankin, 1997). In such 
problems, it is critical to recognize that the stiffness matrix (A7) is referred to the principal coordinate system of the medium, which is not necessarily aligned with the coordinate system of the data. Determination of the angles of rotation relating these two coordinate systems is an essential step of any analysis.

Today, in exploration geophysics, the state-of-the-art for seismic imaging is tilted orthorhombic, with the orthorhombic axes aligned locally with the strike and dip of the subsurface layers. The additional complications arising from the tilt are simply bookkeeping issues.

\section{ACKNOWLEDGMENTS}

The authors thank J.-P. Montagner (IPG, Paris) and Michael Ritzwoller (CU, Boulder) for important insights.

\section{REFERENCES}

Anderson, D. L., 1961, Elastic wave propagation in layered anisotropic media: Journal of Geophysical Research, v.66 (9), p. 2953-2963.

Anderson, D. L., 1966, Recent evidence concerning the structure and composition of the earth's mantle: Physics and Chemistry of the Earth,v. 6, Pergamon Press, Oxford, p. 1-131.

Anderson, D. L., 1989, Theory of the Earth: Blackwell Scientific Publications, Boston, 366 pp.

Anderson, D. L., 2011, Hawaii, Boundary Layers and Ambient Mantle-Geophysical Constraints, Journal of Petrology v. 52 (7-8), p. 1547-1577.

Anderson, D. L., and Dziewonski, A. M., 1982, Upper mantle anisotropy; evidence from free oscillations: Geophysical Journal of the Royal Astronomical Society, v. 69, p. 383-404.

Anderson, D.L. and Natland, J. H., 2014. Mantle updrafts and mechanisms of oceanic volcanism, in press: Proceedings National Academy of Sciences, v. , p 
Babuska, V., and Cara, M., 1991. Seismic Anisotropy in the Earth, Springer, 219 pp.

Backus, G. 1962, Long-wave elastic anisotropy produced by horizontal layering: Journal of Geophysical Research, v. 67(11), p. 4427-4440.

Beghein, C., and Trampert. J., 2003, Probability density functions for radial anisotropy: implications for the upper $1200 \mathrm{~km}$ of the mantle: Earth and Planetary Science Letters, v. 217, p. 151-162.

Boschi L, and Dziewonski, A. M., 2000, Whole Earth tomography from delay times of $P, P c P$, and $P K P$ phases: lateral heterogeneities in the outer core or radial anisotropy in the mantle?: Journal of Geophysical Research, v. 105(13), p. 675-96.

Carcione, J., 2001, Wave fields in real media: Wave propagation in anisotropic, anelastic and porous media: Pergamon Press, New York, 390 pp.

Chen, M., and Tromp, J. 2007, Theoretical and numerical investigations of global and regional seismic wave propagation in weakly anisotropic earth models, Geophysical Journal International, v. 168(3), p. 1130-1152, doi:10.1111/j.1365-246X.2006.03218.x.

Collins, J. A., Wolfe, C. J., and Laske, G., 2012, Shear-wave splitting at the Hawaiian hotspot from the PLUME land and ocean bottom seismometer deployments: Geochemistry Geophysics Geosystems, v. 13, p. 1-15, doi:10.1029/2011GC003881.

Crampin, S. and Peacock, S., 2008, A review of the current understanding of shear-wave splitting and common fallacies in interpretation: Wave Motion, 45, 675-722.

Crampin, S. and Gao, Y., 2013, The New Geophysics: Terra Nova, 25, 173-180.

Dziewonski, A. M., and Anderson, D. L., 1981, Preliminary reference Earth model: Physics of the Earth and Planetary Interiors, v. 25, p. 297-356.

Ekstrom, G. and Dziewonski, A.M., 1998, The unique anisotropy of the Pacific upper mantle: Nature, v. 394, p. 168-172. 
Farra, V. and Psencik, I., 2010. Coupled $S$ waves in inhomogeneous weakly anisotropic media using firstorder ray tracing: Geophys. J. Int., v. 180, p. 405-417.

Gung, Y., Panning, M., and Romanowicz, B., 2003, Global anisotropy and the thickness of continents: Nature, v. 422(6933), p. 707-711.

Jech J., and Psencik, I., 1989, First-order perturbation method for anisotropic media: Geophysical Journal International, v. 99, p. 369-376.

Katzman, R., Zhao, L., and Jordan, T. H., 1998, High-resolution, two-dimensional vertical tomography of the central Pacific mantle using $\mathrm{ScS}$ reverberations and frequency-dependent travel times?, Journal of Geophysical Research, v. 103(B8), p. 17,933-17,971.

Kawakatsu, H., Kumar, P., Takei, Y., Shinohara, M., Kanazawa, T., Araki, E., and Suyehiro, K., 2009, Seismic evidence for sharp lithosphere-asthenosphere boundaries of oceanic plates: Science, v. 324 (5926), p. 499-502.

Keller, W. R., Anderson, D. L., and Clayton, D. W., 2000, Resolution of tomographic models of the mantle beneath Iceland: Geophysical Research Letters, v. 27, p. 3993-3996.

Lei, J. and Zhao, D., 2006, A new insight into the Hawaiian plume: Earth and Planetary Science Letters, v. 241 , p. $438-453$.

Love, A. E. H., 1928, A Treatise on the mathematical theory of elasticity, reprinted 2008 by Dover Publications, New York.

Montagner, J.-P., 2007, Upper mantle Structure: Global isotropic and anisotropic tomography: Ch. 16 in Treatise on Geophysics, Vol. 1: Seismology and Structure of the Earth (Eds. A.M. Dziewonski and B. Romanowicz), Elsevier, p. 559-590.

Montagner, J- P. and Anderson, D. L, 1989a, Petrological constraints on seismic anisotropy: Physics of the Earth and Planetary Interiors, v. 54, p. 82-105. 
Montagner, J.-P., and Anderson, D. L, 1989b, Constrained reference mantle model: Physics of the Earth and Planetary Interiors, v. 58, p. 205-227.

Montagner, J.-P. and Jobert, N, 1988, Vectorial Tomography - II. Application to the Indian Ocean, Geophysical Journal, v. 94, 309-344.

Montagner J.-P., and Nataf, H. C., 1986, On the inversion of the azimuthal anisotropy of surface waves: Journal of Geophysical Research, v. 91, p. 511-520.

Montagner J.-P., and Nataf, H. C., 1988, Vectorial Tomography - I. Theory: Geophysical Journal, v. 94, 295-307.

Montagner, J.-P., and Tanimoto, T., 1991, Global upper mantle tomography of seismic velocities and anisotropies: Journal of Geophysical Research, v. 96, p. 20337-20351.

Montelli R., Nolet, G., Dahlen, F. A., Masters, G., Engdahl, R., and Hung, S., 2004, Finite frequency tomography reveals a variety of mantle plumes: Science, v. 303, p. 338-343.

Nataf, H.-C., Nakanishi, I., and Anderson, D. L., 1986, Measurements of Mantle Wave Velocities and Inversion for Lateral Heterogeneities and Anisotropy, Part III: Inversion: Journal of Geophysical Research, v. 91(B7), p. 7261-7307.

Nettles, M., and Dziewoński, A. M., 2008, Radially anisotropic shear velocity structure of the upper mantle globally and beneath North America, Journal of Geophysical Research, v. 113, p. B02303, doi:10.1029/2006JB004819.Panning, M. and Romanowicz, B., 2004, Inferences on flow at the base of Earth's mantle based on seismic anisotropy: Science, v. 303 (5656), p. 351-353.

Park, J., 1996, Surface waves in layered anisotropic structures, Geophysical Journal International, v. 126(1), p. 173-183, doi:10.1111/j.1365---246X.1996.tb05276.x.

Park, J., and Levin, V. 2002, Seismic Anisotropy: Tracing Plate Dynamics in the Mantle, Science, v. 296(5567), p. 485-489, doi:10.1126/science.1067319. 
Regan, J., and Anderson, D. L., 1984. Anisotropic models of the upper mantle: Physics of the Earth and Planetary Interiors, v. 35, p. 227-263

Romanowicz, B., 2003, Global mantle tomography: progress status in the last 10 years, Annual Review of Geophysics and Space Physics, v. 31(1), p. 303-328.

Romanowicz, B. and Snieder, R., 1988, A new formalism for the effect of lateral heterogeneity on normal modes and surface waves-II. General anisotropic perturbation: Geophysical Journal, v. 93, p. 91 99.

Savage, M. K., 1999, Seismic anisotropy and mantle deformation: What have we learned from shear wave splitting?, Reviews of Geophysics., v. 37(1), p. 65-106.

Shapiro, N. M., and Ritzwoller, M. H., 2002, Monte---Carlo inversion for a global shear velocity model of the crust and upper mantle, Geophysical Journal International, v. 151(1), p. 88-105, doi:10.1046/j.1365-246X.2002.01742.x.

Smith, M. L. and Dahlen, F. A., 1973, The azimuthal dependence of Love and Rayleigh wave propagation in a slightly anisotropic medium, Journal of Geophysical Research, v. 78(17), p. 3321-3333.

Song'T. R. A. and Kawakatsu'H., 2012, Subduction of oceanic asthenosphere: Evidence from sub-slab seismic anisotropy, Geophysical Research Letters, v. 39, L17301-L17306, DOI: $10.1029 / 2012$ GL052639.

Song, X. and Richards, P. G., 1996, Seismological Evidence for Differential Rotation of the Earth's inner core, Nature, v. 382, p. 221-224.

Soldati, G., Boschi, L., and Piersanti, A., 2003, Outer core density heterogeneity and the discrepancy between PKP and PcP travel time observations, Geophysical Research Letters, v. 30(4), doi:10.1029/2002GL016647.

Stoneley, R., 1949, The seismological implications of aeolotropy in continental structure: 
Monthly Notices, Royal Astronomical Society, Geophysical Supplement, v. 5, p. 222232.

Tanimoto, T., and Anderson, D. L., 1985, Lateral heterogeneity and azimuthal anisotropy of the upper mantle: Love and Rayleigh waves 100-250 sec: Journal of Geophysical Research., v. 90, p. 1842-1858.

Thomsen, L., 1986, Weak Elastic Anisotropy, Geophysics, v. 51(10), p. 1954-1966.

Thomsen, L., 2014 Understanding Seismic Anisotropy in Exploration and Exploitation, the SEG/EAGE Distinguished Instructor Short Course \#5 Lecture Notes, $2^{\text {nd }}$ Edn,. Society of Exploration Geophysicists, Tulsa .

Thomsen, L, and Dellinger, J., 2003, On shear-wave triplication in polar-anisotropic media: Journal of applied Geophysics, v. 54, p. 289-296.

Trampert J., and Woodhouse, J. H., 2003, Global anisotropic phase velocity maps for fundamental mode surface waves between 40 and 150 s: Geophysical Journal International, v. 154(1), p. 154-165.

Trampert J., and van Heijst, H. J., 2002, Global azimuthal anisotropy in the transition zone: Science, v. 296 (5571), p. 1297-1299.

Tromp, J., 2001, Inner-core anisotropy and rotation, Annual Review of Earth and Planetary Sciences, v. $29,47-69$.

Tsvankin, I., 1997, Anisotropic parameters and P-wave velocity for orthorhombic media: Geophysics, v. 62, p. 1292-1309.

Tsvankin, I., 2012, Seismic Signatures and Analysis of Reflection Data in Anisotropic Media, $3^{\text {rd }}$ Edn.: Society of Exploration Geophysicists, Tulsa.

Tsvankin, I., Gaiser, J., Grechka, V., van der Baan, M., and Thomsen, L., 2010, Seismic anisotropy in exploration and reservoir characterization: An overview, Geophysics, v. 75(5), p. 75A15-75A29. 
Vinnik L, Breger L, and Romanowicz B., 1998, Anisotropic structures at the base of the mantle, Nature, v. $393,564-567$.

Voigt, W., 1910, Lehrbuch der Kristallphysik: Teubner, Leipzig.

Wang, N., Montagner, J.-P., Fichtner, A.,and Capdeville, Y., 2013, Intrinsic versus extrinsic seismic anisotropy: The radial anisotropy in reference Earth models, Geophysical Research Letters, v. 40, P. 4284-4288, doi:10.1002/grl.50873, 2013.

West, M., W. Gao, and S. Grand (2004), A simple approach to the joint inversion of seismic body and surface waves applied to the southwest U.S., Geophysical Research Letters, v. 31, p. L15615, doi:10.1029/2004GL020373.

West, J. D., Fouch, M. J., Roth, J. B. and Elkins-Tanton, L.T., 2009, Geophysical detection of lithospheric delamination beneath the Great Basin: Nature Geoscience, v.2, p. 439-444.

Wolfe, C. J., Solomon, S. C., Laske, G., Collins, J. A., Detrick, R. S., Orcutt, J. A., Bercovici,, D. and Hauri. E. H., 2009, Mantle shear-wave velocity structure beneath the Hawaiian hot spot: Science, v. 326, p. 1388-1390, doi:10.1126/science.1180165.

Wolfe, C. J., Solomon, S. C., Laske, G., Collins, J. A., Detrick, R. S., Orcutt, J. A., Bercovici,, D. and Hauri. E. H., 2011, Mantle P-wave velocity structure beneath the Hawaiian hot spot: Earth and Planetary Science Letters, v. 303, p. 267-280, doi:10.1016/j.eps1.2011.01.004. 